\title{
Method for Direct Measurement of Cosmic Acceleration by 21-cm Absorption Systems
}

\author{
Hao-Ran Yu, ${ }^{1,2}$ Tong-Jie Zhang, ${ }^{1, *}$ and Ue-Li Pen ${ }^{3, \dagger}$ \\ ${ }^{1}$ Department of Astronomy, Beijing Normal University, Beijing 100875, China \\ ${ }^{2}$ Kavli Institute for Astronomy and Astrophysics, Peking University, Beijing 100871, China \\ ${ }^{3}$ Canadian Institute for Theoretical Astrophysics, \\ University of Toronto, Toronto, M5S 3H8, Ontario, Canada
}

(Dated: Received August 1, 2014; published - 00, 0000)

\begin{abstract}
So far there is only indirect evidence that the Universe is undergoing an accelerated expansion. The evidence for cosmic acceleration is based on the observation of different objects at different distances, and requires invoking the Copernican cosmological principle, and Einstein's equations of motion. We examine the direct observability using recession velocity drifts (Sandage-Loeb effect) of $21 \mathrm{~cm}$ hydrogen absorption systems in upcoming radio surveys. This measures the change in velocity of the same objects separate by a time interval and is a model-independent measure of acceleration. We forecast that for a CHIME-like survey with a decade time span, we can detect the acceleration of a $\Lambda$ CDM Universe with $5 \sigma$ confidence. This acceleration test requires modest data analysis and storage changes from the normal processing, and cannot be recovered retroactively.
\end{abstract}

PACS numbers: 98.80.Es 95.36.+x

Introduction. - One of the biggest mysteries in physics is the purported accelerated expansion of the Universe. This suggests that gravity on the largest scales is not an attractive force, but for the Universe at large is repulsive. Strong mysteries require strong and preferably direct evidence, and to date the inference of acceleration is indirect. Direct evidence would require the measurement of velocities of objects at cosmological distances at different epochs, and determining an increase in recession velocity for the same objects.

Current indirect inferences require the application of Einstein's equation combined with a Copernican principle. The Copernican principle underlies the assumption of large scale homogeneity of the cosmos, which enables the general solution of the complex, non-linear Einstein equations. The indirect inferences include dynamical measurements of the gravitational evolution of structure[1,2], or kinematic measurements using luminosity or angular diameter distances [3-7].

They are indirect because they measure different objects at multiple distances (redshifts) in the Universe at an instant of time on earth, and the acceleration is inferred by assuming a homogeneous cosmological model and an equation of motion. The direct modelindependent physical acceleration is by definition the velocity change over a time interval between two measurements. A direct probe of the cosmic acceleration is the Sandage-Loeb (SL) effect [8, 9], suggested by Sandage to measure change of redshift of galaxies, and by Loeb to measure the drift in the Ly $\alpha$ forest. The latter proposal motivates the construction of the high-resolution CODEX $^{1}$ (COsmic Dynamics and EXo-earth experi-

\footnotetext{
* tjzhang@bnu.edu.cn

$\dagger$ pen@cita.utoronto.ca

1 http://www.iac.es/proyecto/codex/
}

ment) spectrogragh on E-ELT ${ }^{2}$ (European Extremely Large Telescope), to measure the precise redshift of Lya over a two decade interval. The CODEX group provide a full design for observing the SL signal and a prediction of the statistical error of the SL signal [10]. It is also shown that the effect has the potential to better constrain dark energy [11-13]. This ambitious study primarily searches for cosmic deceleration and jerk at redshift $z \gtrsim 2$, and is not sensitive to direct acceleration measurements. For a direct measurement of acceleration, a lower redshift is preferable, when the Universe is actually accelerating.

In the radio domain, the $21 \mathrm{~cm}$ line in the hydrogen absorption systems are the most promising candidates to detect the acceleration. In damped Ly $\alpha$ absorption systems (DLA), the radio spectrum is also substantially absorbed by the neutral hydrogen (HI) hyperfine structure which has the rest frame wavelength of approximately $21 \mathrm{~cm}$ or frequency of $1420 \mathrm{MHz}$. DLA's are defined as having $\mathrm{HI}$ column density greater than $2 \times 10^{20}$ $\mathrm{cm}^{-2}$. This density results in substantial self-shielding, and empirically is also the minimum column to house cold $21 \mathrm{~cm}$ absorbing gas in the cold neutral medium (CNM). Treated as $21 \mathrm{~cm}$ hydrogen absorption systems, the measurements are readily accessible over the cosmic accelerating redshift using ground based radio telescopes. Their advantages include: (1) the intrinsic line width is narrow because absorption is dominated by cold absorbers at $T<80 \mathrm{~K}$. (2) collecting area is cheap in the redshifted $21 \mathrm{~cm}$ band. (3) a new generation of telescopes is being constructed in the relevant bands. The best direct constraint on the acceleration of the Universe to date is based on observing these systems [14] over more than a decade. These errors are still three orders of magnitude larger than a prediction from a $\Lambda \mathrm{CDM}$ (cold dark matter

\footnotetext{
${ }^{2}$ http://www.eso.org/public/teles-instr/e-elt.html
} 
with a cosmological constant $\Lambda$ ) Universe and overwhelm the real signal.

Large numbers of $21 \mathrm{~cm}$ absorption systems in the purported accelerating regime of the Universe will be detected by wide-sky radio surveys like PARKES ${ }^{3}$, underconstruction ASKAP[15], CHIME $^{4}$, GBT-multibeam ${ }^{5}$ and proposed BAOBAB[16], BAOradio[17], BINGO[18], $\mathrm{CARPE}^{6}, \mathrm{MeerKAT}^{7}, \mathrm{SKA}^{8}$ and Tianlai ${ }^{9}$ [19], etc. In the remainder of this letter we will estimate how this tiny SL effect could be extracted statistically within a decade from instruments that are being constructed or proposed, in many cases for the purpose of more precise indirect measurements of dark energy.

Quantifying the direct acceleration. - The acceleration $\dot{v}$ of a given object at redshift $z$ is given by its redshift drift

$$
\dot{v}=c \dot{z} /(1+z),
$$

where over-dots denote derivatives respect to observation time $\mathrm{d} / \mathrm{d} t$ and $c$ is the speed of light. Here the redshift drift $\dot{z}$ is linearly related to the Hubble parameter $H(z)$ at the object's redshift $z$

$$
\dot{z}=(1+z) H_{0}-H(z),
$$

where Hubble constant $H_{0}$ is the current expansion rate $H(z=0)$ and $H(z)$ is given by the specified cosmological model. In a concordance $\Lambda$ CDM Universe for instance, objects with redshift $z \lesssim 2.5$ are accelerating and their acceleration $\dot{v}$ is by order of $0.1 \mathrm{~cm} \mathrm{~s}^{-1} \mathrm{yr}^{-1}$. To measure this minuscule velocity difference one needs to subtract off proper accelerations of the observer. It can be precisely measured by pulsar timing in the Galactic frame $[20,21]$ and by proper motion of the extra-galactic radio sources in the cosmological frame [22]. Even without the observations of the proper accelerations and without full-sky coverage, one can still solve the cosmic and local accelerations by a moment analysis over the sky.

Feasibility. - Assuming a $\Lambda$ CDM Universe, the $21 \mathrm{~cm}$ absorption systems in the redshift range $0<z<2.5$ are accelerating, which corresponds to the frequency range from $1420 \mathrm{MHz}$ to $406 \mathrm{MHz}$. CHIME $(0.8<z<2.5)$ and PARKES $(0<z<1.0)$-like surverys are suitable to be devised to scan the radio sources in NRAO VLA Sky Survey $\left(\mathrm{NVSS}^{10}\right)$, looking for possible $21 \mathrm{~cm}$ absorption systems. Here we discuss a decade-long-survey by a CHIME-like telescope as an example.

\footnotetext{
3 phased array feed project on parkes, http://www.atnf.csiro. $\mathrm{au} / \mathrm{research} / \mathrm{multibeam} /$

${ }^{4}$ http://CHIMExperiment.ca

5 http://gbt800mb.pbworks.com/

${ }^{6}$ http://www.phys.washington.edu/users/mmorales/carpe/

7 http://www.ska.ac.za/meerkat/

8 http://www.skatelescope.org

9 http://tianlai.bao.ac.cn

10 http://www.cv.nrao.edu/nvss/
}

If the survey scans the northern-hemisphere of the sky, it is about $60 \%$ area of NVSS coverage. NVSS contains a catalog of almost 2 million discrete sources, with flux densities $F$ brighter than about $2.5 \mathrm{mJy}$ at $1.4 \mathrm{GHz}$. The CHIME-like survey will be sensitive to absorbers in the 1.2 million NVSS sources in its field of view. CHIME's frequency coverage requires $\nu<800 \mathrm{MHz}$, where the sources are typically brighter by $\nu^{-0.7}$. We choose a lower flux limit of $4.5 \mathrm{mJy}$, where the source counts are well understood, and which is more than $17 \sigma$ above the thermal noise limit. For a rough estimation of the redshift distribution of the observed $21 \mathrm{~cm}$ absorption systems, we may adopt a NVSS redshift distribution $n_{\mathrm{R}}(z)$ (e.g. eq(26) in [23]) and neutral hydrogen cloud redshift distribution $n_{\mathrm{HI}}(z)$ (the density of optical damped Ly $\alpha$ systems is $14 \%$ per unit redshift [24]). If those two components are uncorrelated and randomly distributed throughout the sky, the resulting redshift distribution of $21 \mathrm{~cm}$ absorption systems will be $n_{21 \mathrm{~cm}}(z)=n_{\mathrm{H} \mathrm{I}}(z) \int_{z}^{\infty} n_{\mathrm{R}}\left(z^{\prime}\right) \mathrm{d} z^{\prime}$. We plot $n_{21 \mathrm{~cm}}(z)$ and $n_{\mathrm{R}}(z)$ in the upper panel of Fig.1.

The signal-to-noise ratio $(S / N)$ equals to the flux $F$ of the radio sources divided by the error of the measurement $\Delta F$. For a dual polarized system,

$$
S / N=F / \Delta F=F \sqrt{2 \Delta \nu \Delta t} / \mathrm{SEFD},
$$

where $\Delta \nu, \Delta t$ are the line width and integration time respectively, and SEFD is the system equivalent flux density. For CHIME, we adopt $\mathrm{SEFD}=25 \mathrm{Jy}$.

The line width $\Delta \nu=u_{\text {width }} \nu / c$, where $u_{\text {width }}$ is the equivalent width of the HI absorber, and $\nu$ is the frequency of the absorber. The properties of $21 \mathrm{~cm}$ absorbers are primarily derived from followup studies of optical absorbers. The discovery rate from the cross correlation is a lower bound on the expect number of absorbers, since high column density systems in the CNM may systematically obscure potential background optical sources. There are only 3 blind radio detections, and a survey may discover more systems which are optically obscured. They would likely be cold and at high column density. For sensitivity purposes, we treat all sources as $u_{\text {width }}$ of $2 \mathrm{~km} \mathrm{~s}^{-1}[24,25]$. In practice, sources typically have an equivalent $u_{\text {width }}$ of $2 \mathrm{~km} \mathrm{~s}^{-1}$, and an actual line structure which is varied and diverse, and beyond of the scope of this paper. Wider structures potentially reduce the survey sensitivity. On the other hand, radio searches will preferentially find cold and narrow line systems, potentially increasing the sensitivity beyond the values adopted here. Thus the line width $\Delta \nu \simeq 9.3 \mathrm{kHz}$. The integration time $\Delta t=t_{\text {survey }} \tau$, where $t_{\text {survey }}$ is the total time survey duration and $\tau=\lambda / 2 \pi D$ is the fractional time that an object on the celestial equator transits the field of view of the telescope ${ }^{11}$. For CHIME,

11 Objects at higher latitude have greater $\tau$. If objects are uniformly distributed on the sky and $\lambda \ll D$, we have a boost factor $b=\pi / 2$ on integration time or $(S / N)^{2}$. We take this factor into account in the calculation. 


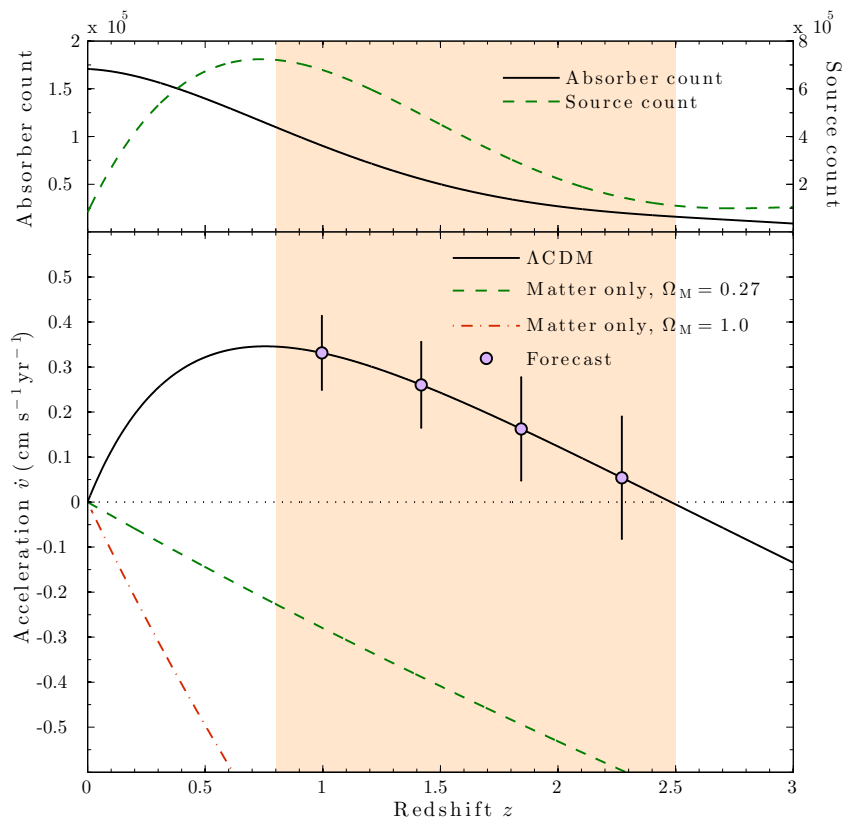

FIG. 1. (color online.) (top:) Northern-hemisphere number counts per unit redshift $(\mathrm{d} N / \mathrm{d} z)$ for $21 \mathrm{~cm}$ absorption systems $f_{21 \mathrm{~cm}}$ in black solid line, and NVSS radio sources $f_{\mathrm{QSO}}$ in (green) dashed line. (bottom:) Velocity drift forecast by $21 \mathrm{~cm}$ absorption systems by CHIME-like telescope assuming a concordance $\Lambda$ CDM Universe. The observation span is 10 years. The black solid line is the theoretical prediction by a $\Lambda$ CDM Universe, while the predictions of matter-only universes $\Omega_{\mathrm{M}}=0.27, \Omega_{\mathrm{M}}=1$, which are decelerating, are shown in (green) dashed line and (red) dash-dotted line. CHIME's observable redshift range $0.8<z<2.5$ is shown by (orange-) shaded regions in both panels of the figure.

the diameter $D$ of each cylinder is $20 \mathrm{~m}$ and the observational wavelength $\lambda$ is $21 \mathrm{~cm}(1+z)$. For a ten-year survey $\left(t_{\text {survey }}=3.16 \times 10^{8} \mathrm{sec}\right), \Delta t \simeq 5.28 \times 10^{5}(1+z) \mathrm{sec}$ at the equator. Substituting $\Delta \nu$ and $\Delta t$ into Eq.(3), we get $\Delta F=0.252(1+z)^{-1 / 2}$ mJy.

In order to construct the velocity and acceleration estimator we write $v=v_{0}+\dot{v} t+\eta$ and $\eta$ is Gaussian noise: $\left\langle\eta(t) \eta\left(t^{\prime}\right)\right\rangle=\sigma_{\eta}^{2} \delta\left(t-t^{\prime}\right)$. We have the velocity estimator $E_{v}=\left(1 / t_{\text {survey }}\right) \int v \mathrm{~d} t$ and acceleration estimator $E_{\dot{v}}=\left(12 / t_{\text {survey }}^{3}\right) \int v t \mathrm{~d} t$ such that $\left\langle E_{v}\right\rangle=v$ and $\left\langle E_{\dot{v}}\right\rangle=\dot{v}$ (integrals go from $-t_{\text {survey }} / 2$ to $t_{\text {survey }} / 2$ ). Their errors can be estimated by $\sigma_{v}^{2}=\left\langle E_{v}^{2}\right\rangle-\left\langle E_{v}\right\rangle^{2}$ and $\sigma_{\dot{v}}^{2}=\left\langle E_{\dot{v}}^{2}\right\rangle-\left\langle E_{\dot{v}}\right\rangle^{2}$. By integration we can get $\sigma_{v}=\sigma_{\eta} t_{\text {survey }}^{-1 / 2}$ and $\sigma_{\dot{v}}=2 \sqrt{3} \sigma_{\eta} t_{\text {survey }}^{-3 / 2}$, meaning that $\sigma_{\dot{v}}=2 \sqrt{3} \sigma_{v} t_{\text {survey }}^{-1}$ : error is enlarged as we are requiring the time derivative information. The forecast of $\sigma_{v}$ also depends on various of factors like the actual line structure and sampling quantization. For a rough estimation, treating line structure as Gaussian with standard deviation $\sigma$, a 16-level quantization (with frequency bin $0.34 \sigma$ ) gives at least $98.8 \%$ quantization efficiency [26], and thus $340 \mathrm{~m} \mathrm{~s}^{-1}$ sampling will be enough. Other effects will become limiting factors as the quantization efficiency approaches unity when we do a even finer frequency sampling. According to the central limit theorem, The error of the velocity estimator $\sigma_{v}$ is given by $u_{\text {width }}(S / N)_{\text {bin }}^{-1}$ so we can as well forecast $\sigma_{\dot{v}}$, where in general,

$$
(S / N)_{\text {bin }}^{2}=\iint_{F_{\min }}^{\infty}\left(\frac{b F^{2}}{\Delta F^{2}} n_{\mathrm{HI}}(z) \int_{z}^{\infty} n_{\mathrm{R}}\left(F, z^{\prime}\right) \mathrm{d} z^{\prime}\right) \mathrm{d} F \mathrm{~d} z,
$$

is the cumulative $(S / N)^{2}$ in one redshift bin. In relevant frequency bands, all $\mathrm{d} n_{\mathrm{R}} / \mathrm{d} F$ have similar profile [27] and using any one of those gives nearly the same result (we use $\mathrm{d} n_{\mathrm{R}} / \mathrm{d} F$ at $0.61 \mathrm{GHz}$ [27], as it is the main contribution of background sources), so we do not assume any redshift dependence on the flux distribution. Thus the inner integration in Eq.(4) can be written as $\int_{z}^{\infty} n_{\mathrm{R}}\left(z^{\prime}\right) \mathrm{d} z^{\prime}$.

Binning all the data into four redshift bins from redshift 0.8 to 2.5 , we plot the binned velocity drift forecast $\dot{v}(z)$ with error bars in the lower panel of Fig.1.

For the assumed $\Lambda$ CDM Universe, we can detect the acceleration with $5.1 \sigma$ confidence. If taken as an indirect test of acceleration, this same data in combination with Einstein's equations and homogeneity could be used to exclude matter-only universes with $\Omega_{\mathrm{M}}=0.27$ and $\Omega_{\mathrm{M}}=$ 1 with $12.5 \sigma$ and $29.4 \sigma$ confidence respectively.

Discussion. - CHIME and other experiments are constructed to make precision indirect measurements of dark energy. Modest real-time analysis changes could allow the direct detection of cosmic acceleration. The data would need to be recorded at sufficient spectral resolution, better than $340 \mathrm{~m} \mathrm{~s}^{-1}$, corresponding to $800 \mathrm{~Hz}$, which is not originally planned. The frequency channelization cost is FFT based, and increasing from a spectral resolution of 300 to $10^{5}$ doubles the FFT cost. Spatial computational costs are in principle unchanged, but there could be additional overhead costs for the larger resulting data sets. Frequency stability of $10^{-11}$ over a decade is required, which is straightforward on decade time scales with GPS-rubidium clocks, but also needs to be built into the system from the beginning. Foreground contamination would not be a problem in these very narrow frequency bands: one expects the spatial-frequency mixing of foregrounds for the filled aperture experiment to be $(\delta \lambda / \lambda)^{2}$, which leaves the foregrounds far below the thermal noise.

The substantial improvement from previous estimates [14] arises from the persistent daily observations of every system, and the 10,000 fold increase in number of targets from the rapid all sky survey. We have applied a conservative cut on source detections of more than $17 \sigma$. The number of targets could be larger if compensation for false detection rates is allowed. Due to the paucity of known $21 \mathrm{~cm}$ absorbers, and absence of accurate blind surveys, the actual $21 \mathrm{~cm}$ detection rate could be different from our assumptions [28]. Astronomical complexities, including multiple absorption features within systems, variations in optical depths and line width, are not well characterized and have been neglected. This could 
lead to sensitivity changes, either positive or negative. We stress that the incremental effort needed for this experiment is minimal, and well worth the effect, even if only to characterize the large number of absorption systems.

Detection of the acceleration by promising $21 \mathrm{~cm}$ absorption systems needs redshift below 2.5 which corresponds to the frequency from $1420 \mathrm{MHz}$ to $406 \mathrm{MHz}$. Velocity drift data on frequencies lower than $406 \mathrm{MHz}$ $(z>2.5)$ will show decelerations and are no longer the most direct evidence of accelerating expansion. If CHIME is scheduled for completion in 2015, a tenyear campaign could result in direct detections in 2025 . The above estimation has substantial room for a further improvement as we can also include the southernhemisphere through SKA and potential southern hemisphere CHIME-like telescope. Completing the entire sky completely removes the acceleration bias in Galactic and cosmological frame. Moreover, by using all frequencies up to $1420 \mathrm{MHz}$, it will also broaden the redshift range, as there are still lots of absorber counts at $0<z<0.8$ with obvious acceleration (see Fig.1). If combined with a southern hemisphere "CHIME"-like experiment with a decade cadence, the cosmic acceleration measurement would be improved to $\mathrm{a} \sim 8 \sigma$ confidence level.

Our proposed experiment observes only the velocity changes of same single objects over a time interval, and thus the acceleration measure is geometrical and does not require any assumptions of homogeneity, isotropy and Einstein equations. However, in order to test the dark energy or Lemaitre-Tolman-Bondi (LTB) models, we still need to include isotropy, homogeneity and metric assumptions [29-31]. On the other hand, without these assumptions, adequate accuracy of the measurement also enables us to test any anisotropic cosmic acceleration or inhomogeneity of the Universe non-parametrically.

Conclusions. - We have estimated the sensitivity of upcoming radio experiments to a direct cosmic acceleration search. We conclude that this detection may be possible with a CHIME-like and subsequent telescopes, if appropriate real-time data processing modifications were made. A direct detection of cosmic acceleration bypasses Copernican cosmological principle and Einstein Equation assumptions normally required to infer the most mysterious property of the Universe: acceleration.

Acknowledgments. - We thank Avi Loeb, Keith Vanderlinde, Nissim Kanekar, Jeremy Darling and Mark Halpern for helpful comments and discussions. This work was supported by the National Science Foundation of China (Grants No. 11173006), the Ministry of Science and Technology National Basic Science program (project 973) under grant No. 2012CB821804, and the Fundamental Research Funds for the Central Universities.
[1] J. P. Ostriker and P. J. Steinhardt, Nature (London)377, 600 (1995).

[2] The ISW effect is a dynamical consequence of dark energy. For the lasted analysis of ISW, see Planck Collaboration et al., ArXiv e-prints (2013), 1303.5079.

[3] A. G. Riess et al., Astron. J. 116, 1009 (1998).

[4] S. Perlmutter et al., Astrophys. J.517, 565 (1999).

[5] P. de Bernardis et al., Nature (London)404, 955 (2000).

[6] Planck Collaboration, ArXiv e-prints (2013), 1303.5076.

[7] D. J. Eisenstein et al., Astrophys. J.633, 560 (2005), arXiv:astro-ph/0501171.

[8] A. Sandage, Astrophys. J.136, 319 (1962).

[9] A. Loeb, Astrophys. J. Lett. 499, L111 (1998), arXiv:astro-ph/9802122.

[10] J. Liske et al., Mon. Not. R. Astron. Soc. 386, 1192 (2008), 0802.1532.

[11] P.-S. Corasaniti, D. Huterer, and A. Melchiorri, Phys. Rev. D75, 062001 (2007), arXiv:astro-ph/0701433.

[12] Z. Li, K. Liao, P. Wu, H. Yu, and Z.-H. Zhu, Phys. Rev. D88, 023003 (2013), 1306.5932.

[13] S. Yuan, S. Liu, and T.-J. Zhang, ArXiv e-prints (2013), 1311.1583.

[14] J. Darling, Astrophys. J. Lett. 761, L26 (2012), 1211.4585.

[15] J. Darling, E. P. Macdonald, M. P. Haynes, and R. Giovanelli, Astrophys. J.742, 60 (2011), 1108.4011.

[16] J. C. Pober et al., Astron. J. 145, 65 (2013), 1210.2413.

[17] R. Ansari et al., Comptes Rendus Physique 13, 46 (2012), 1209.3266 .
[18] R. A. Battye et al., ArXiv e-prints (2012), 1209.1041.

[19] X. Chen, International Journal of Modern Physics Conference Series 12, 256 (2012), 1212.6278.

[20] N. L. Zakamska and S. Tremaine, Astron. J. 130, 1939 (2005), arXiv:astro-ph/0506548.

[21] M. H. Xu, G. L. Wang, and M. Zhao, Astron. Astrophys. 544, A135 (2012).

[22] O. Titov, S. B. Lambert, and A.-M. Gontier, Astron. Astrophys. 529, A91 (2011), 1009.3698.

[23] G. de Zotti, M. Massardi, M. Negrello, and J. Wall, Astron. Astrophys. Rev. 18, 1 (2010), 0908.1896.

[24] A. M. Wolfe, E. Gawiser, and J. X. Prochaska, Ann. Rev. Astron. Astrophys. 43, 861 (2005), arXiv:astro$\mathrm{ph} / 0509481$.

[25] A. M. Wolfe, F. H. Briggs, and M. M. Davis, Astrophys. J.259, 495 (1982).

[26] A. R. Thompson, J. M. Moran, and G. W. Swenson, Jr., Interferometry and Synthesis in Radio Astronomy, 2nd Edition (, 2001).

[27] J. J. Condon, Astrophys. J.287, 461 (1984).

[28] F. B. Abdalla, C. Blake, and S. Rawlings, Mon. Not. R. Astron. Soc. 401, 743 (2010), 0905.4311.

[29] J.-P. Uzan, C. Clarkson, and G. F. R. Ellis, Physical Review Letters 100, 191303 (2008), 0801.0068.

[30] C. Quercellini, L. Amendola, A. Balbi, P. Cabella, and M. Quartin, Phys. Rep. 521, 95 (2012), 1011.2646.

[31] D. H. Weinberg et al., Phys. Rep. 530, 87 (2013), 1201.2434. 